\title{
The pulvinar nucleus and antidepressant treatment: dynamic modeling of antidepressant response and remission with ultra-high field functional MRI
}

\author{
Christoph Kraus $\mathbb{B}^{1} \cdot$ Manfred Klöbl ${ }^{1} \cdot$ Martin Tik $\mathbb{D}^{2} \cdot$ Bastian Auer $^{3} \cdot$ Thomas Vanicek $^{1} \cdot$ Nicole Geissberger $^{2}$.

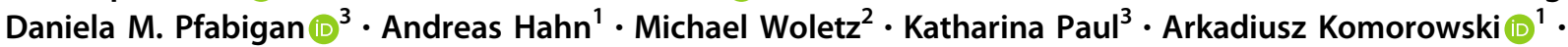 \\ Siegfried Kasper ${ }^{1} \cdot$ Christian Windischberger $^{2} \cdot$ Claus Lamm $^{3} \cdot$ Rupert Lanzenberger (1) ${ }^{1}$
}

Received: 8 June 2017 / Revised: 5 October 2017 / Accepted: 27 October 2017 / Published online: 8 February 2018

(c) The Author(s) 2018. This article is published with open access, corrected publication February 2018

\begin{abstract}
Functional magnetic resonance imaging (fMRI) successfully disentangled neuronal pathophysiology of major depression (MD), but only a few fMRI studies have investigated correlates and predictors of remission. Moreover, most studies have used clinical outcome parameters from two time points, which do not optimally depict differential response times. Therefore, we aimed to detect neuronal correlates of response and remission in an antidepressant treatment study with $7 \mathrm{~T}$ fMRI, potentially harnessing advances in detection power and spatial specificity. Moreover, we modeled outcome parameters from multiple study visits during a 12-week antidepressant fMRI study in 26 acute (aMD) patients compared to 36 stable remitted (rMD) patients and 33 healthy control subjects (HC). During an electrical painful stimulation task, significantly higher baseline activity in aMD compared to HC and rMD in the medial thalamic nuclei of the pulvinar was detected ( $p=0.004$, FWE-corrected), which was reduced by treatment. Moreover, clinical response followed a sigmoid function with a plateau phase in the beginning, a rapid decline and a further plateau at treatment end. By modeling the dynamic speed of response with fMRI-data, perigenual anterior cingulate activity after treatment was significantly associated with antidepressant response $(p<0.001$, FWE-corrected). Temporoparietal junction (TPJ) baseline activity significantly predicted non-remission after 2 antidepressant trials $(p=0.005$, FWE-corrected). The results underline the importance of the medial thalamus, attention networks in MD and antidepressant treatment. Moreover, by using a sigmoid model, this study provides a novel method to analyze the dynamic nature of response and remission for future trials.
\end{abstract}

The original version of this article was revised: The author list was updated to list the authors names correctly. Full information regarding corrections made can be found in the erratum/correction article for this article.

Electronic supplementary material The online version of this article (https://doi.org/10.1038/s41380-017-0009-x) contains supplementary material, which is available to authorized users.

Rupert Lanzenberger

rupert.lanzenberger@meduniwien.ac.at

1 Neuroimaging Labs, Department of Psychiatry and Psychotherapy, Medical University of Vienna, Vienna, Austria

2 Center for Medical Physics and Biomedical Engineering, Medical University of Vienna, Vienna, Austria

3 Social, Cognitive and Affective Neuroscience Unit, Faculty of Psychology, University of Vienna, Vienna, Austria

\section{Introduction}

First-line medication of major depression (MD) consists of antidepressant treatment with selective serotonin-reuptake inhibitors (SSRIs) [1, 2]. After non-responsive treatment in the first trial a second trial with an antidepressant with a differential mechanism of action such as a serotoninnoradrenaline reuptake inhibitor (SNRI) is often performed. After both sequential treatments in total about 30-60\% achieve remission, leaving about $70-40 \%$ of all patients depressed [3, 4]. For medication-naive patients, estimation of future response and remission remains open to clinical judgments [5]. To obtain biological markers of treatment effects, studies investigated blood-oxygen-level dependent (BOLD) signal changes upon antidepressant treatment with fMRI. In the beginning, most of these studies investigated correlates of response [6], only recently fMRI-studies 
focused on remission [7]. While neurobiological response markers may be of interest for drug development or clinical research, predictive markers of remission have the potential to be clinically applicable, by allowing patients and physicians to anticipate who will maximally improve before treatment.

A recent fMRI antidepressant trial investigated predictive markers of remission [8]. In this study, dorsolateral prefrontal cortex (dlPFC) activity in a Go/NoGo response inhibition task significantly predicted remission, whereas higher post-treatment dlPFC activity was associated with non-remission. A prior investigation failed to detect baseline differences between remitter and non-remitter using a Stroop Color-Word paradigm [9]. These previous fMRI studies on remission to antidepressant therapy applied lower field strengths at $3 \mathrm{~T}$ [8] and $1.5 \mathrm{~T}$ [9].

Other neurobiological substrates of depression are the anterior and medial cingulate cortex as well as the insula. Areas that can be activated by various salient stimuli like electrical painful stimulation [10]. In a pilot study we showed that $7 \mathrm{~T}$ fMRI had advantageous BOLD responses to transcutaneous electrical stimulation as compared to $3 \mathrm{~T}$ in brain areas associated with pain processing [11]. Based on the neuronal activation pattern of painful stimuli and our pilot study, we conducted an antidepressant treatment study at $7 \mathrm{~T}$ with the aim of assessing treatment effects in order to investigate correlates of response and discover predictors of remission. Furthermore, we aimed to improve response assessment by modeling clinical outcome parameters from all available study visits. Applying dynamic response modeling we aim to predict response and remission in an explanatory whole-brain approach, without limitation to a priori defined regions of interest. Based on the previous study [10] and our pilot study, we hypothesized that anterior and midcingulate cortex as well as insular, prefrontal and basal ganglia functionality would exhibit significant treatment effects.

\section{Methods}

\section{Participants and treatment}

All participants gave oral and written informed consent to the study protocol. Subjects were compensated financially for their participation. The study protocol and all study related procedures were approved by the Ethics Committee of the Medical University (EK 103/2011) of Vienna and registered at clinicaltrials.gov (NCT01477203). Study duration was from November 2011 to September 2016. For inclusion and exclusion criteria see supplementary methods.

All medication free acute (aMD) patients underwent an open label, flexible dose, unblinded, but standardized antidepressant treatment for 12 weeks with control visits with possible dosage adjustment every two weeks. fMRI-1 was at week 0 and fMRI-2 was scheduled 12 weeks later. As first line treatment, all aMD patients received escitalopram oxalate for six weeks. A starting dosage of $5 \mathrm{mg}$ was allowed for better tolerance for a maximum of 5 days, afterwards flexible escitalopram dosages between $10-20 \mathrm{mg}$ were given according to clinical judgment by study psychiatrists. Down-titration to 15 or $10 \mathrm{mg}$ was allowed, if 20 mg were not tolerated due to side effects.

If acute patients did not respond to the first-line antidepressant after 6 weeks, defined by at least 50\% Hamilton Depression Rating Scale (HAM-D 24 ) reduction compared to first visit, they were switched to a second trial with venlafaxine extended release $75-150 \mathrm{mg}$ or mirtazapine 30-60 $\mathrm{mg}$, if venlafaxine was not tolerated. If venlafaxine or mirtazapine was previously not tolerated, escitalopram $10-20 \mathrm{mg}$ was allowed to continue, even if patients did not respond after 6 weeks. The second trial treatment was administered for another six weeks with control visits until fMRI-2 ( $11=$ inclusion, $v 2=$ fMRI 1 and week 0 of treatment, $\mathrm{v} 3=$ week $2, \mathrm{v} 4=$ week $4, \mathrm{v} 5=$ week $6, \mathrm{v} 6=$ week $8, \mathrm{v} 7=$ week 12 and fMRI-2, v8 $=$ follow up). All three groups were measured 12 weeks apart for fMRI-1 and fMRI-2.

\section{Outcome parameters}

All patients were assessed by psychiatrists with at least 3 years of experience in clinical psychological testing at every visit with $\mathrm{HAM}-\mathrm{D}_{24}$, Hamilton Anxiety Rating Scale (HAM-A), Beck Depression Index (BDI), and Clinical Global Impression Scale (CGI). Response (-50\% HAM$\mathrm{D}_{24}$ compared to visit 1) was assessed at every visit. Remission was assessed at visits 5 (week 6) and 7 (= fMRI-2, week 12) and defined as $<8$ on the HAM- $\mathrm{D}_{24}$ scale, which was chosen as a conservative cutoff to minimize residual symptoms. Healthy controls and remitted (rMD) subjects were tested with the same questionnaires at baseline visit, fMRI-1 and fMRI-2, as well as at end-ofstudy examination.

\section{fMRI analyses}

The entire pain paradigm and image preprocessing are detailed in the supplementary material and in the pilot studies [11, 12]. Single-subject analysis was conducted using the General Linear Model approach as implemented in SPM12 (http://www.fil.ion.ucl.ac.uk/spm/). For each possible anticipation and stimulation condition, a regressor was modeled. Nuisance regressors included 6 realignment parameters, 4 white matter, and 1 ventricles confounding signal.

To test group differences and hence treatment effects between fMRI scans a $3 \times 2$ repeated measures ANOVA 
(rmANOVA) was calculated in SPM12 with group as between-subject and scan as within-subject factor contrasting pain vs. no-pain and expect vs. unsure conditions. This analysis was corrected for sex, age, and an index for the individually calibrated pain stimulation intensity (1-10) at the scan day (= painful intensity-non painful intensity). Post-hoc $t$-tests between every group and scan were performed.

Based on visual inspection of HAM-D ${ }_{24}$ scores across all 8 visits, a sigmoid course was suggested. Therefore, response over visits was fitted in a sigmoidal model with scaling and offset parameters for time between visits and HAM-D 24 scores with following parameters:

$$
\sigma(t)=\frac{a}{1+e^{b t+c}}+d
$$

whereby $a$ represented the amplitude between highest and lowest HAM-D 24 score (i.e., maximum change in HAM-D 24 score), $b$ was the time scaling, i.e., the speed of transition between upper and lower plateau phase, $c$ modeled the temporal shift of the curve, and $d$ was the lowest HAM-D ${ }_{24}$ value allowed. The parameter $b$ was most important for the current study since it reflects the dynamic change of HAM- ${ }_{24}$ score over time (i.e., the speed how fast a patient experiences changes in HAM-D 24 score). If model fitting with all available data points lead to implausible results (e.g., reduction of the transition between the plateau phases to a step function), responsible outliers were identified and removed (maximum one per subject). Fitting was accomplished using the MATLAB "fit" function (2013b, The MathWorks, Inc., Natick, Massachusetts, United States, Curve Fitting Toolbox 3.4.) with the "least absolute residuals" method.

To model the dynamic response to antidepressant treatment over 12 weeks in the group of currently depressed patients, the speed of transition values $(b)$, i.e., the slopes of the dynamic response function, were used in a regression model with pain, no-pain, expect and unsure contrasts in the pain paradigm in SPM 12 correcting for sex, age, and pain stimulation indices. Imaging results were correlated with escitalopram or venlafaxine plasma values (supplementary methods).

Finally, we assessed predictability or correlates of remission between remitted and non-remitted acute depressed patients after 12 weeks antidepressant treatment. To evaluate the association between the slope of the HAM-D ${ }_{24}$ curve and BOLD responses, a linear regression analysis was performed in SPM12 across the group of currently depressed patients. To test for significant differences in this association between remitter and non-remitter, a voxel-wise ANOVA was carried out [13]. Covariates of non-interest in the model were age, sex, and stimulation thresholds (difference between pain stimuli and non-pain stimuli).

All results were corrected applying the family-wise error rate $(\mathrm{FWE})$ at $p<0.05$ voxel-level or cluster-level (vFWE or cFWE, as indicated below), the latter following $p<0.001$ uncorrected voxel-level.

\section{Results}

For subject details and clinical outcomes please see Fig. 1, Table 1 and supplementary results.

\section{Neuroimaging results}

\section{Treatment effects ANOVA}

Acute depressed subjects exhibited significantly different activation (i.e., hyperactivation) in the no pain vs. baseline contrast peaking in the left thalamic pulvinar nuclei, the ventral posterior nucleus, mammillary body and medial dorsal nucleus at baseline $\left(F_{2,9}=14.6\right.$, cFWE $p=0.004$,

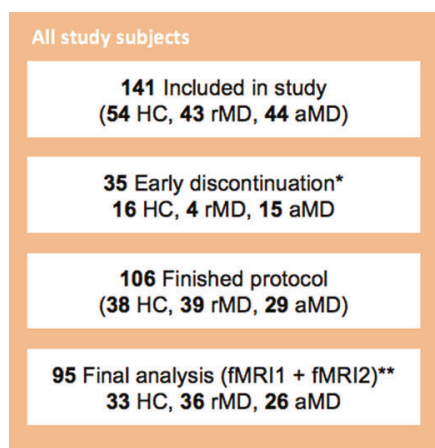

Fig. 1 Subject numbers and outcomes. Numbers of all study subjects are given on the left side. * Early discontinuation reasons are listed at Table S3 in supplemental material. **Numbers correspond to final dataset, reasons for exclusion from data-analyses are given in Table S4. Outcomes after 12 week (wk)-flexible dose antidepressant

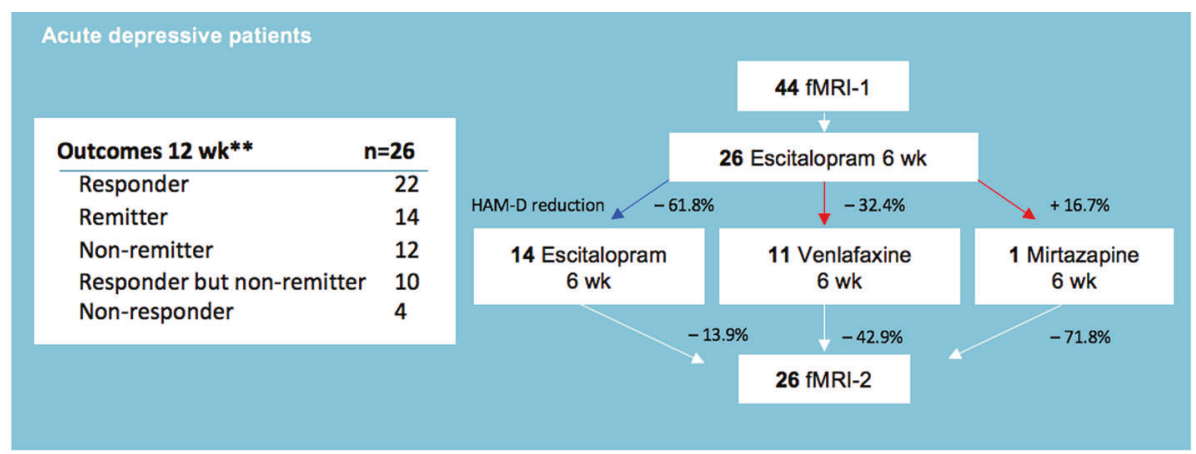

treatment with escitalopram, venlafaxine, and mirtazapine are presented on the right side. Responder are defined by $50 \%$ Hamilton Depression Rating Scale $\left(\mathrm{HAM}-\mathrm{D}_{24}\right)$ reduction to baseline or more, remitter by $\mathrm{HAM}-\mathrm{D}_{24}<8$. Percentages represent reduction of $\mathrm{HAM}_{-} \mathrm{D}_{24}$ 


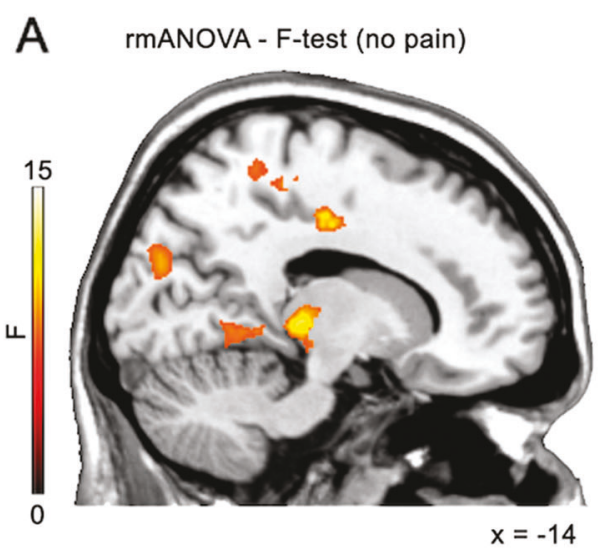

\section{total sample}
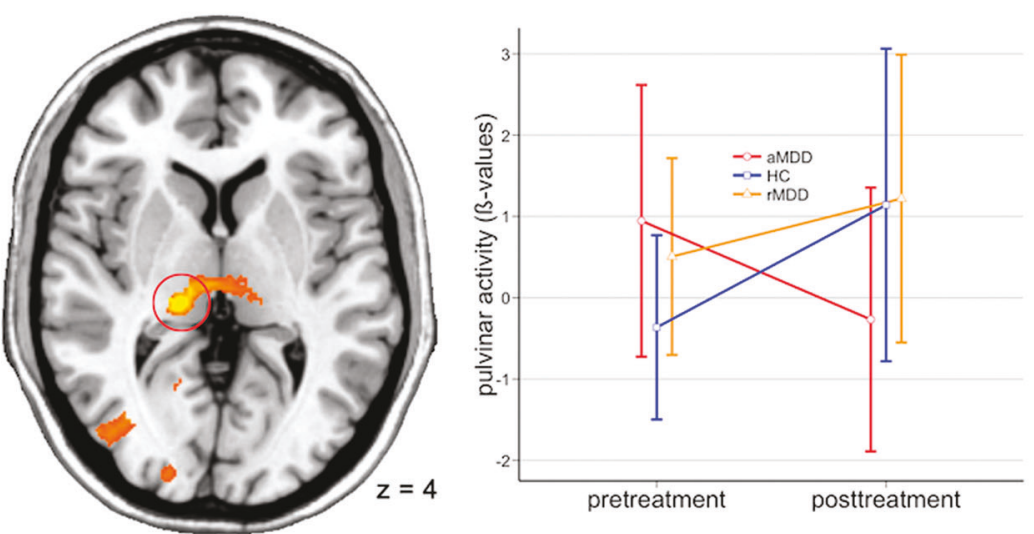

B T-test (no pain)
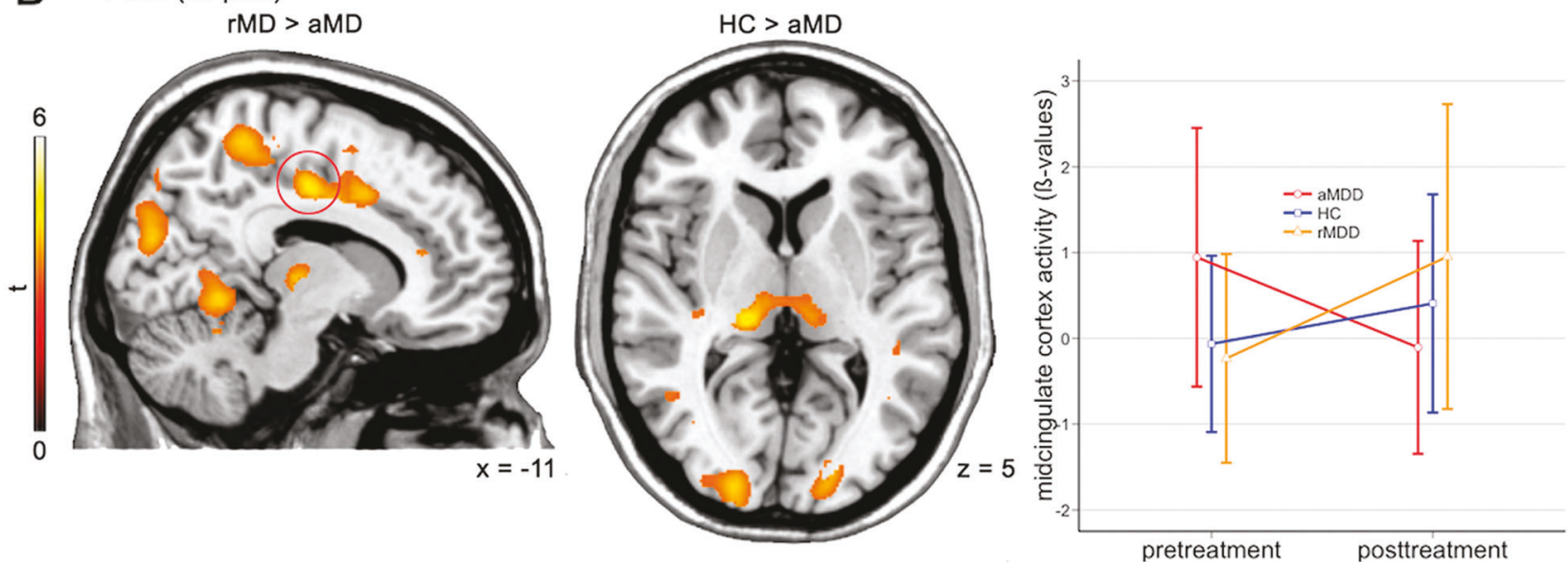

Fig. 2 Reversal of thalamic hyperactivity after antidepressant treatment. Repeated measures analysis of variance (rmANOVA) comparisons between fMRI-1 and fMRI-2 within healthy controls (HC), remitted depressed patients (rMD), and acute depressed patients (aMD). Acute patients were treated with escitalopram or venlafaxine for 12 weeks. a, $F$-test results depicts elevated activation of thalamic pulvinar nuclei in acute patients, which reversed to baseline values of
$\mathrm{HC}$ after treatment. Activations represents difference in $F$-test between fMRI-2 vs. fMRI-1. b, Post-hoc $t$-tests revealed elevated activity in midcingulate cortex. Activations represents difference in $t$-test between fMRI-2 vs. fMRI-1. Errorbar-plots represent activation values of red circled-clusters, which survived family-wise error correction (FWE, cluster-level, $p<0.05$ ). Left is left. Results are depicted at $p<0.001$ uncorrected voxel level see Fig. 2, Figure S4 and for statistics Table S2). Compared to $\mathrm{rMD}(t=4.3$, cFWE $p=0.019)$ and $\mathrm{HC}(t=5.4$, cFWE $p=0.001)$ hyperactivity in aMD was reduced to values of $\mathrm{HCs}$ at baseline after 12 weeks of treatment. An additional significant cluster was obtained in the right superior occipital cortex/cuneus $\left(F_{2,9}=9.4\right.$, df $=2 / 9$, cFWE $\left.p=0.003\right)$. A similar pattern, which did not survive statistical correction in the $F$-test was observed in the midcingulate cortex. Here, significantly elevated activation in aMD, which diminished at fMRI-2 with most difference to rMD was observed $(t=4.8$, cFWE $p<0.001)$. Additionally, rMD subjects exhibited higher signal difference between the two scans in the bilateral precuneus, the occipital cortex and the anterior lobe of the cerebellum (see Table S2). To exclude that the effects are solely driven by variability in the healthy control group, we also tested for significant differences only for the aMD group. We observed significantly reduced neuronal activation in the pulvinar and middle cingulate cortex at uncorrected levels $(p<0.001)$. There was no statistical difference in the rmANOVA between rMD subjects and HC (all $p>0.001$ ) in the no pain vs. baseline contrast. All other tested contrast combination yielded negative results (see supplementary results).

\section{Dynamic response regression}

The whole-brain regression analysis in aMD patients with speed of transition values $b$ yielded a significant negative correlation between dynamic $\mathrm{HAM}-\mathrm{D}_{24}$ reduction and perigenual anterior cingulate (pgACC) and medial prefrontal cortex (mPFC) activation $(t=4.9$, cFWE $p<0.001$, see Fig. 3). This result was obtained in 


\section{acute Patients}

A

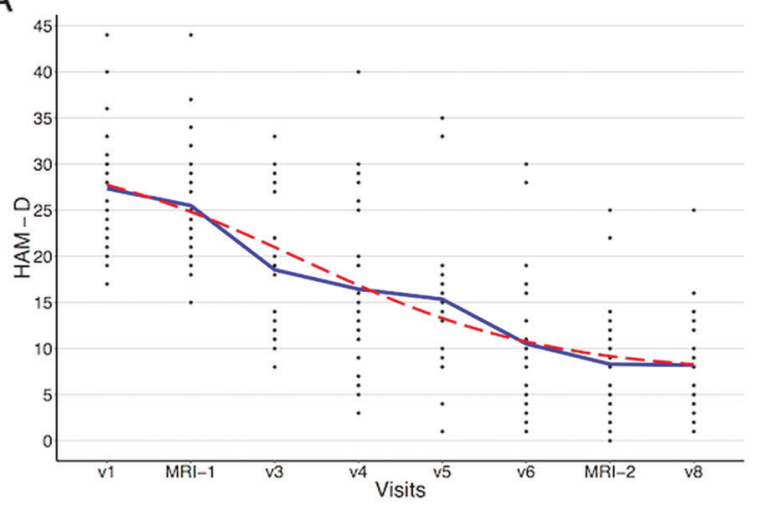

B

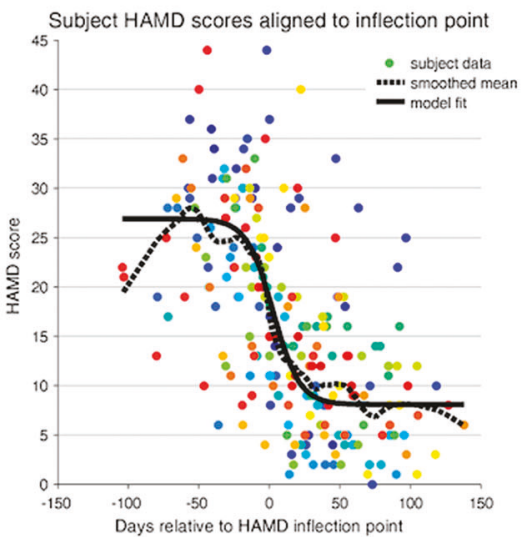

Example subject data
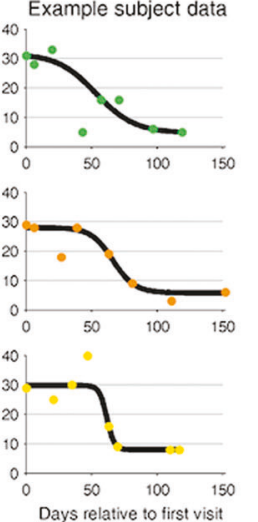

\section{posttreatment}

C
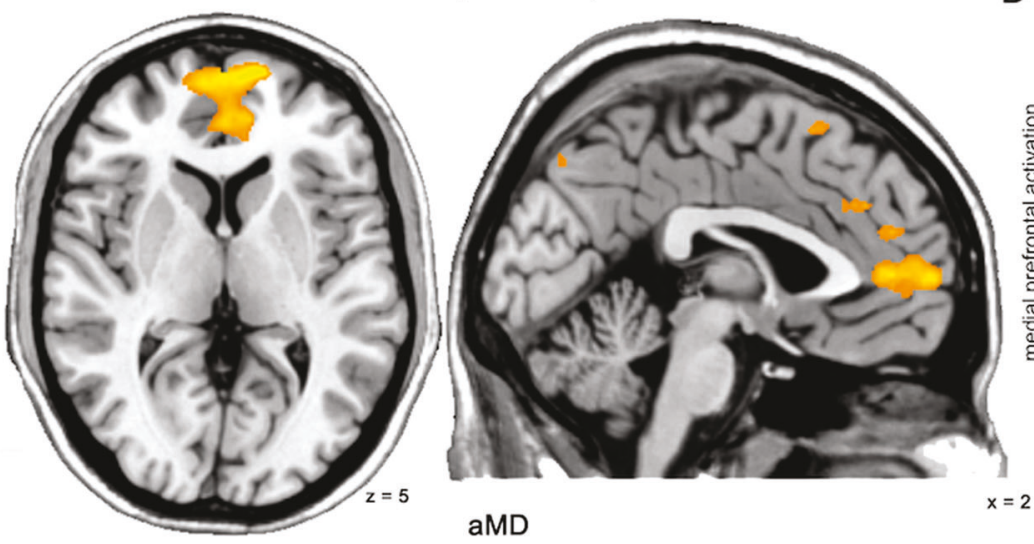

$D$

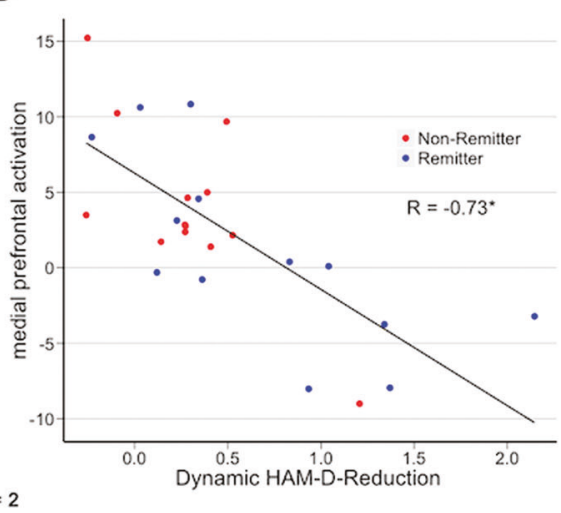

aMD

Fig. 3 Dynamic response to antidepressant treatment. a, Hamilton Depression Rating Scale (HAM-D ${ }_{24}$ ) reduction from baseline visit (v1) to last visit (v8). fMRI-1 and fMRI-2 were 12 weeks apart. The blue line represents means, the red dashed line corresponds to the function in (b). b, HAM-D ${ }_{24}$ reduction was fitted with a general sigmoid model with days between visits and HAM- ${ }_{24}$ scores was fitted for each acute depressed (aMD) patient and aligned at 0 . Colors of single points represent individual patients. c, d The sigmoid function's $b$ (i.e., the

the "unsure no pain" vs. "sure no pain" contrast at fMRI2 , there were no other significant results surviving cFWE-correction in other contrasts or fMRI-1. Simply using the difference between HAM- ${ }_{24}$ scores from MRT-1 and MRT-2 did not result in a significant association with BOLD signal changes $(p>0.001$ uncorrected). By plotting the activation values of the cluster against $b$ (corrected for age, sex, and difference in stimulation thresholds), we observed that all but one nonremitter exhibited higher activation after treatment, i.e., in these patients this region was not downregulated after treatment. However, this was not specific to non-remitter, since activation with remitters overlapped.

The mean $\beta$-values of the cluster in the $\mathrm{pgACC} / \mathrm{mPFC}$ significantly correlated with escitalopram $(r=0.77, p=$ $0.005)$, but not with venlafaxine plasma values $(r=0.05$, $p=0.89$, see Figure S3). slope of the HAM- $\mathrm{D}_{24}$ reduction) was used in a regression model and yielded a significant negative correlation $(p<0.001$, FWE, clusterlevel) between dynamic HAM-D ${ }_{24}$ reduction and perigenual cingulate and medial prefrontal activation at fMRI-2. Non-Remitter (= HAM$\mathrm{D}_{24}>8$ ) exhibited higher activation after treatment. Activations represents t-tests between remitter and non-remitter at fMRI-2. * represents Pearson correlation coefficient at $p<0.05$

\section{Prediction of remission}

Activation in the right temporoparietal junction (TPJ) at fMRI-1 significantly separated remitter from non-remitter in the expect vs. unsure stimuli $(t=4.1, \mathrm{cFWE} p=0.005$, see Fig. 4a). This cluster had an accuracy of 58\%, sensitivity of $41.6 \%$ and specificity of $71.4 \%$. While remitting patients increased their activation between fMRI-1 and fMRI-2, non-remitter had a decrease of activation at fMRI-2 (Fig. 4a). The difference between fMRI-1 and fMRI-2, i.e., effects of treatment between remitter and non-remitter, was associated with significantly higher activation in the left orbitofrontal cortex in non-remitter $(t=4.7$, vFWE $p=$ $0.034)$. The cluster is located lateral and inferior to the cluster obtained by the response regression analysis in all subjects (accuracy $=54 \%$, sensitivity $=50 \%$, specificity $=$ $57 \%$ ). Activation plots show that non-remitter exhibited 
Fig. 4 Non-remitter failed to upregulate temporoparietal junction after treatment . a, Acute depressed patients who remitted (aMDDrem) after 12 weeks antidepressant treatment had significantly more activity at fMRI-2 in the right temporoparietal junction in the expect vs. unsure contrast (asterisk in a represents $p=$ 0.005 , FWE cluster-level).

Remission was defined conservatively by HAM-D ${ }_{24}<8$. Activation represents $t$-tests between remitter and nonremitter at fMRI-2. b, Nonremitter exhibited significantly increased activity between fMRI-1 and fMRI-2 in the no pain contrast $(* *$ represents $p=$ 0.034, FWE voxel-level). Activation represents $t$-tests between remitter and nonremitter between fMRI-2 and fMRI-1
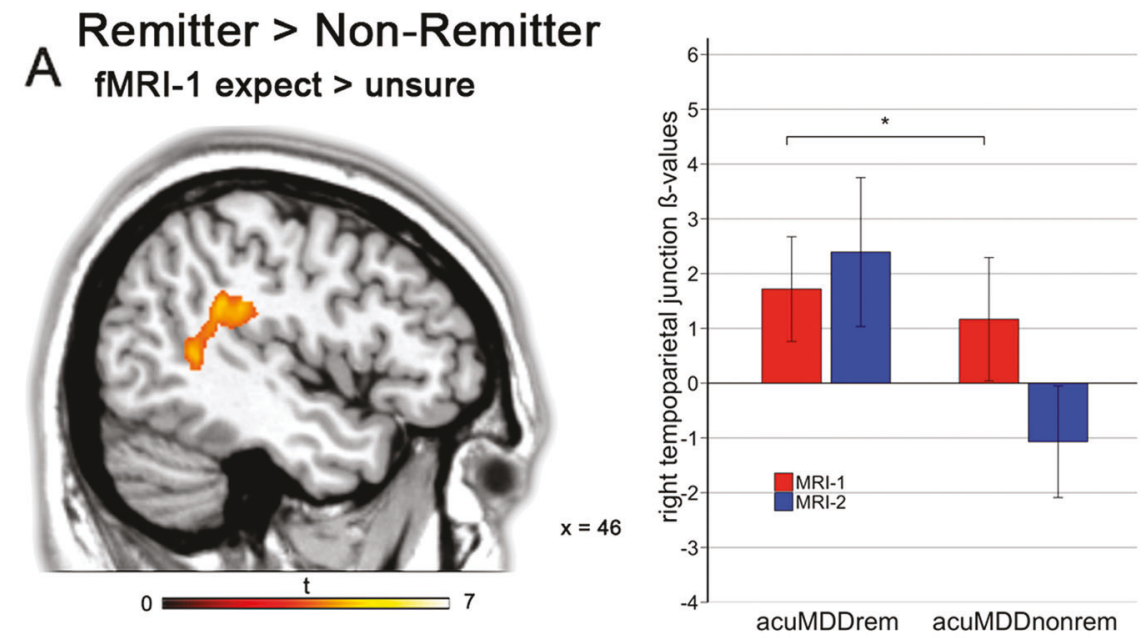

B Non-Remitter > Remitter (fMRI-2) - (fMRI-1):

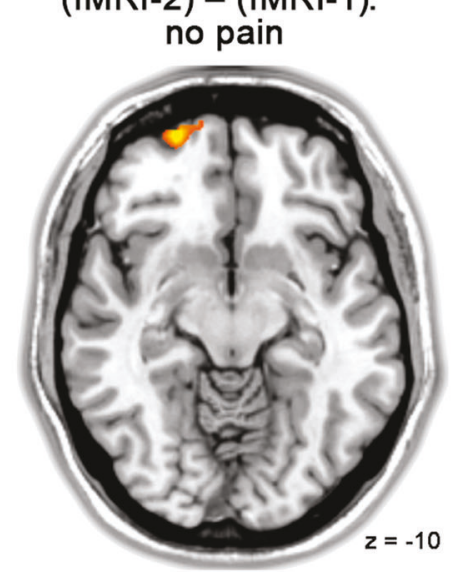

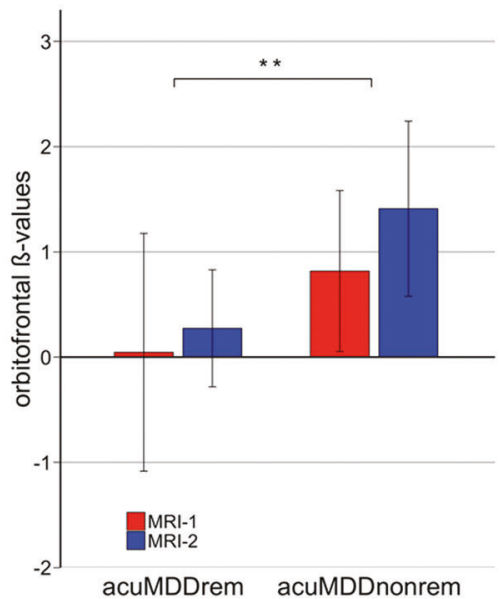

higher baseline activation and increased orbitofrontal activity at fMRI-2 in comparison to remitting patients (Fig. 4b). An ANOVA (time point $\times$ group) between remitting and non-remitting MD without the sigmoid model did not yield significant results (all $p>0.001$, uncorr.).

\section{Discussion}

In this 12-week, open-label, antidepressant fMRI study we identified reduction of elevated baseline thalamic activity in the pulvinar and medial thalamus as significant antidepressant treatment effect. These data indicate that antidepressant treatment reverses elevated activity in regions of a major thalamocortical network with strong connections to the limbic system [14]. Furthermore, optimized detection of response and remission was achieved by mapping the dynamic speed of individual treatment response with a sigmoid function. Thereby, aberrant frontal and anterior cingulate activity were confirmed as important correlates of antidepressant response [6, 15-17]. Finally, we detected that remission was associated with higher pretreatment TPJ activity. Together, these results speak for reversal of hyperactivity in regions within salient and attention networks as an important antidepressant effect.

Our data are not in accordance with those resulting from a prior pain fMRI study [10]. It is striking that the main results were obtained in the no-pain vs. baseline condition, even though pain yielded expected activations (Figure S2). Heterogeneity in the depression group with mixed antidepressant treatments at fMRI-2 or a ceilingeffect at $7 \mathrm{~T}$, which might not be present in lower field strengths during physiologically stronger pain-stimuli, could explain missing results in the pain vs. no-pain contrasts. Between group heterogeneity or measurement time points might not be the main driver of the pulvinar result, since pulvinar changes were detected in aMD only as well. Moreover, these studies differ in regard to the field strengths used, treatment regimens, and comparison groups. Even though $7 \mathrm{~T}$ might exhibit superiority for fMRIstatistics for most of the brain regions [11] and provide increased spatial specificity [18], this study in concert with 
Table 1 Demographic and clinical characteristics (analyzed sample)

\begin{tabular}{|c|c|c|c|c|c|}
\hline Subjects & $\mathrm{HC}$ & rMD & \multicolumn{2}{|l|}{ aMD } & $p$ \\
\hline$n$ & 33 & 36 & \multicolumn{2}{|l|}{26} & \\
\hline $\operatorname{Sex}(f / m)$ & $20 / 13$ & $23 / 13$ & \multicolumn{2}{|l|}{$19 / 7$} & $0.592^{\mathrm{a}}$ \\
\hline Age $(y)$ & $26.6 \pm 6.8$ & $28.5 \pm 8.2$ & \multicolumn{2}{|l|}{$30.4 \pm 9.7$} & $0.215^{\mathrm{b}}$ \\
\hline Age of onset $(y)$ & - & $22.8 \pm 6.7$ & \multicolumn{2}{|l|}{$21.6 \pm 9.2$} & $0.284^{\mathrm{c}}$ \\
\hline Number of episodes $(n)$ & - & $1.7 \pm 1.4$ & \multicolumn{2}{|l|}{$3.2 \pm 1.6$} & $<0.001^{\mathrm{c}}$ \\
\hline Duration of disease $(y)$ & - & $5.8 \pm 4.8$ & \multicolumn{2}{|l|}{$9.5 \pm 8.8$} & $0.02^{\mathrm{c}}$ \\
\hline Current episode/last episode rMD $(m)$ & - & $9.1 \pm 6$ & \multicolumn{2}{|l|}{$6.8 \pm 12.1$} & $0.128^{\mathrm{c}}$ \\
\hline Previous medication (unclear/yes/no) ${ }^{\mathrm{d}}$ & & $5 / 17 / 13$ & \multicolumn{2}{|l|}{$0 / 14 / 12$} & \\
\hline PT during study (unclear/yes/no) & - & $2 / 4 / 28$ & \multicolumn{2}{|l|}{$4 / 3 / 19$} & \\
\hline PT before study (unclear/yes/no) & - & $8 / 20 / 6$ & \multicolumn{2}{|l|}{$3 / 9 / 13$} & \\
\hline Handedness (r/l) & $32 / 1$ & $34 / 1$ & \multicolumn{2}{|l|}{$25 / 1$} & \\
\hline Scales & & & Pretreatment & Posttreatment & \\
\hline HAM-D 24 & - & $2.3 \pm 2.5$ & $27.3 \pm 6.4$ & $8.3 \pm 5.9$ & \\
\hline HAM-A & - & $2.5 \pm 2.4$ & $20.9 \pm 5.6$ & $6.7 \pm 3.9$ & \\
\hline BDI & - & $4.2 \pm 4.6$ & $21.1 \pm 7.2$ & $7.4 \pm 5.7$ & \\
\hline CGI & - & $1.7 \pm 0.8$ & $5.3 \pm 0.5$ & $3.1 \pm 1.2$ & \\
\hline \multicolumn{6}{|l|}{ Medication during study } \\
\hline Escitalopram (mg) & - & - & - & $13.9 \pm 4.5$ & \\
\hline Venlafaxine (mg) & - & - & - & $98.2 \pm 41.7$ & \\
\hline Mirtazapine (mg) & - & - & - & $30^{\mathrm{e}}$ & \\
\hline Citalopram plasma (ng/ml) & - & - & - & $33.7 \pm 27.3$ & \\
\hline Venlafaxine plasma (ng/ml) & - & - & - & $88.4 \pm 59.2$ & \\
\hline \multicolumn{6}{|l|}{ Painful stimulus intensity } \\
\hline fMRI-1 & $5.6 \pm 5.2$ & $4.5 \pm 3.4$ & \multicolumn{2}{|c|}{$5.1 \pm 2.8$} & $0.567^{\mathrm{b}}$ \\
\hline fMRI-2 & $5.6 \pm 6$ & $4.3 \pm 3.7$ & \multicolumn{2}{|c|}{$5.4 \pm 3.7$} & $0.543^{\mathrm{b}}$ \\
\hline \multicolumn{6}{|l|}{ Non-painful intensity } \\
\hline fMRI-1 & $1.2 \pm 1.1$ & $1.3 \pm 1.1$ & \multicolumn{2}{|c|}{$1.5 \pm 0.8$} & $0.555^{\mathrm{b}}$ \\
\hline fMRI-2 & $1.7 \pm 2.2$ & $1.4 \pm 1.1$ & \multicolumn{2}{|c|}{$1.5 \pm 0.9$} & $0.722^{\mathrm{b}}$ \\
\hline
\end{tabular}

$H C$ healthy control subjects, $r M D$ remitted depressed subjects, aMD acute depressed patients $f M R I-1$ functional magnetic resonance imaging session one, $H A M-D_{24}$ Hamilton Depression Rrating Scale (24 item version), HAM-A Hamilton Anxiety Rating Scale, BDI Beck Depression Index, CGI Clinical Global Impression scale, $f$ female, $m$ male, $y$ years, $m$ months, $r$ right, $l$ left

${ }^{\mathrm{a}} p$-value chi-square test

${ }^{\mathrm{b}} p$-value analysis of variance

${ }^{\mathrm{c}} p$-value $t$-test

${ }^{\mathrm{d}}$ for detiailed medication see supplementary Table S5

enly one patient others show that advantages in group discrimination over lower field strengths might be negligable [19]. For response-prediction markers research, multimodal approaches [20] and machine learning analyses [21] might be more valuable than higher field strengths. For future applications of $7 \mathrm{~T}$ fMRI in depression research, determination of potential confounders such as physiological noise will be particularly important. Increases in signal strength come at the cost of increased physiological noise, whereby regions such as the orbitofrontal cortex with proximal air-tissue boundaries are at risk.
Nevertheless, these results match studies reporting altered pulvinar function in MD and highlight pulvinar and thalamic alterations in the pathophysiologic model of depression. Increased pulvinar BOLD-signals and glucose metabolism in untreated MD are substantiated by three meta-analyses [22-24] and elevated baseline glucose metabolism in the pulvinar was demonstrated to be reduced with remission [25]. Our study adds that increased pulvinar BOLD-signal at baseline was reduced after treatment. Thalamocortical coupling is altered in depression and antidepressant therapy targets this connection [26-28]. 
Antidepressant effects on the pulvinar and the thalamus are substantiated in other fMRI tasks [29-31]. Moreover, thalamocortical connectivity was altered upon ketamine administration [32] and transracial magnetic stimulation to the dorsolateral prefrontal cortex elevated activity in the pulvinar nuclei [33]. These results corroborate reduction of pulvinar hyperactivity by antidepressants, which might not be specific to a pain fMRI paradigm, but a more general effect of antidepressant efficacy.

In the following we discuss potential consequences of altered pulvinar function in MD. The pulvinar nuclei receive the majority of their inputs from the retina and reciprocal connections with striatal, extrastriatal, parietal, temporal, frontal, orbital, cingulate cortices, and the amygdala were described [14, 34]. They provide quick feedback for behaviorally relevant stimuli [35] and monitor attention and/or distraction (reviewed by Pessoa et al. [34]). Irreversible damage to the pulvinar nuclei was associated with visual neglect but also with post-stroke pain [36, 37], which could explain why these results were gathered in a pain-paradigm. Functional changes in the pulvinar, as demonstrated in our study, might contribute to biased acquisition and processing of information, which has been described as important pathophysiological factor in MD [38]. Altered thalamocortical processing was suggested to underlie negativity biases [39], hence, our results might be neurobiological substrates of biased acquisition and retrieval of information, though replication with explicit testing of salience and negativity bias is needed.

The time course of antidepressant response followed a sigmoid function, which has been previously demonstrated [40]. With this optimized model of antidepressant response, we replicated anterior cingulate and ventromedial prefrontal cortex activity as antidepressant treatment response correlate $[6,7,17,41]$. Without dynamic response modeling, no results on response and remission were yielded in this dataset. Elevated pretreatment anterior cingulate activity is most often reported in resting state or facial emotion tasks [6]. Furthermore, the time points of measurement might impact results, since early response might underlie a different neuronal activity as late response [42, 43]. A sigmoid response indicates that individual patients respond with a moderate decline of HAM-D values in the beginning, followed by at a certain period of faster response and an again flatter decline at the end of observations. A steeper decline in the sigmoid function means that the speed of transition from depression to remission is faster. By describing response with a sigmoid function better fits to interindividual variations in speed of response could be gained. But first replication in larger datasets should be performed. Potentially, differential "response constants" could be then determined for pharmacological and nonpharmacological antidepressant treatments. These would decrease the number of required data points and could in future be applied to independent datasets.

Applying the model of dynamic response by multiple study visits, we yielded higher baseline activity in the right temporoparietal junction (TPJ) in remitter. Non-remitter exhibited a decrease of activation after treatment. The TPJ is part of the ventral attention network [44] and usually activated in fMRI studies by reorienting attention to salient stimuli [45]. The TPJ is involved in controlling theory of mind-related abilities [46], which were recently shown to be significantly reduced in MD [47] (although see negative results e.g., ref. [48]). MD patients exhibit a reduced visual arousal in the right TPJ [49]. Treatment elevated TPJhypoactivity towards activity levels of healthy controls [50]. These studies corroborate a role of the TPJ in enabling internal predictions about external sensory events [51], which might be altered in MD. In our study, remission was associated with increased baseline TPJ-activity in relation to levels of activity in non-remitters. Non-remission was associated with increased activity in the left orbitofrontal cortex between fMRI-1 and fMRI-2. The orbitofrontal cortex is prone to susceptibility artifacts, that is why replication of this result is needed. Both these results were not sensitive and specific enough to promote TPJ activity as potential prognostic marker with clinical applicability. While both regions seem to be affected by $\mathrm{MD}$, future studies could look closer into their potential as clinical marker. In general, clinical applicability of task-based fMRI still remains an unresolved issue. Here, machine learning approaches were previously demonstrated to yield higher accuracies with a low minimum sample size [52]. Moreover, resting state analyses outperformed task-based fMRI in a metaanalysis comparing diagnostic classification by machine learning algorithms [21]. Hence, other imaging modalities and analyses strategies than task-based fMRI and the general linear model might in future be more profitable in finding predictive response or remission markers. Nevertheless, we propose that TPJ-activity should be more frequently investigated in MD, since easy accessibility by non-invasive therapies such as TMS could provide rewarding paradigms.

A few factors must be kept in mind when interpreting these findings. Test-retest reliability remains an issue for potential clinical application of fMRI. Several studies with only fair to moderate ICC values in pain paradigms were reported [53], while others find slightly better values [54]. Yet, spatial activation patterns [55] and noise [56] appear to be stable over measuring time-points. Since we detected significant differences in aMD patients only, we do not conclude that the pulvinar result is solely driven by heterogeneity potentially present between measurements or groups. Second, we did not correct for potential effects of psychotherapy, which might be a limiting factor of the 
current study design. However, previous evidence demonstrated differential effects of remission to psychotherapy in the anterior insula and the subgenual cingulate [57, 58], while no effects on the thalamus were demonstrated. The potential influence in our sample, given low numbers of current psychotherapy, might be small, but future studies should control for psychotherapy. A third limitation might be the lack of placebo control. While all applied antidepressants were demonstrated to be superior to placebo $[59,60]$, this does not rule out unwanted placebo effects in this study. Placebo control in fMRI treatment trials is currently infrequently applied [8, 61, 62]. For translation of pharmacological fMRI findings to clinic placebo-controlled validation might be necessary. In the end, generalization of predictive markers derived from studies as ours to clinical populations remains very hard, because inclusion and exclusion criteria constrain the heterogeneity of major depression in clinical settings.

\section{Conclusion}

This antidepressant fMRI treatment study in patients with MD applying a pain-paradigm revealed elevated activity in the medial thalamus, especially in the pulvinar nuclei, which declined after treatment. Thalamic and pulvinar activity are a major contributor to depression pathophysiology. Moreover, we optimized response assessment by modeling the dynamic speed of response using a sigmoidal model with fMRI-data and corroborated the importance of anterior cingulate function in remission. Moreover, our results hint towards temporoparietal junction activity as a potential correlate of non-remission, but confirmation is needed. The main conclusion of this study is that pulvinar activity has a key function in depression and antidepressant treatment, while optimized modeling of response and remission might be a valuable method for future clinical depression trials.

Acknowledgements This research was supported by the intramural grant 'Multimodal Neuroimaging in Clinical Neurosciences-Assessment of neurobiological markers for psychiatric disorders' of the research cluster between the Medical University of Vienna and the University of Vienna, by the grant 'Interdisciplinary Translational Brain Research Cluster (ITHC) with highfield MR' from the Federal Ministry of Science, Research, and Economy (BMWFW), Austria, a grant by the Austrian Science Fund (FWF, No. KLI 516) to R. Lanzenberger and a grant by the Austrian National Bank (OeNB, Anniversary Fund No. 14577) to S. Kasper. We thank G.S. Kranz, S. Ganger, R. Seiger, J. Losak, R. Sladky, M. Küblböck, A. Hoffmann, A. Hummer, I.L. Stürkat, K. Paul, A. Wucherer, A. Grahl, C. Siegl, D. Fraissl, D. Willinger, M. Hubinger, J. Hass for methodological or technical support and D. Winkler, M. Spies, P. Baldinger, A. Höflich, J. Unterholzner, M. Godbersen, L, Schwarz, L. Silberbauer, P. Köck, O. Mahlberg, C. Winkler, R. Hoffmann, M. Svagr, and V. Rotter for clinical support with the study.

\section{Compliance with ethical standards}

Conflict of interest SK received grants/research support, consulting fees and/or honoraria within the last 3 years from Angelini, AOP Orphan Pharmaceuticals AG, AstraZeneca, Eli Lilly, Janssen, KRKAPharma, Lundbeck, Neuraxpharm, Pfizer, Pierre Fabre, Schwabe and Servier. RL received travel grants and/or conference speaker honoraria from AstraZeneca, Lundbeck A/S, Dr. Willmar Schwabe GmbH, Orphan Pharmaceuticals AG, Janssen-Cilag Pharma GmbH, and Roche Austria GmbH. TV received travel grants and compensation for workshop participation from Pfizer and Eli Lilly and speaker honoraria from Eli Lilly. CK has received travel grants from Roche Austria $\mathrm{GmbH}$ and AOP Orphan. The remaining authors declare that they have no conflict of interest.

Open Access This article is licensed under a Creative Commons Attribution-NonCommercial-ShareAlike 4.0 International License, which permits any non-commercial use, sharing, adaptation, distribution and reproduction in any medium or format, as long as you give appropriate credit to the original author(s) and the source, provide a link to the Creative Commons license, and indicate if changes were made. If you remix, transform, or build upon this article or a part thereof, you must distribute your contributions under the same license as the original. The images or other third party material in this article are included in the article's Creative Commons license, unless indicated otherwise in a credit line to the material. If material is not included in the article's Creative Commons license and your intended use is not permitted by statutory regulation or exceeds the permitted use, you will need to obtain permission directly from the copyright holder. To view a copy of this license, visit http://creativecommons. org/licenses/by-nc-sa/4.0/.

\section{References}

1. Kasper S, Moller HJ, Montgomery SA, Zondag E. Antidepressant efficacy in relation to item analysis and severity of depression: a placebo-controlled trial of fluvoxamine versus imipramine. Int Clin Psychopharmacol. 1995;9:3-12.

2. Bauer M, Whybrow PC, Angst J, Versiani M, Moller HJ. World Federation of Societies of Biological Psychiatry Task Force on Treatment Guidelines for Unipolar Depressive D. World Federation of Societies of Biological Psychiatry (WFSBP) Guidelines for Biological Treatment of Unipolar Depressive Disorders, Part 2: Maintenance treatment of major depressive disorder and treatment of chronic depressive disorders and subthreshold depressions. World J Biol Psychiatry. 2002;3:69-86.

3. Rush AJ, Trivedi MH, Wisniewski SR, Stewart JW, Nierenberg AA, Thase ME, et al. Bupropion-SR, sertraline, or venlafaxineXR after failure of SSRIs for depression. N Engl J Med. 2006;354:1231-42.

4. Trivedi MH, Rush AJ, Wisniewski SR, Nierenberg AA, Warden D, Ritz L, et al. Evaluation of outcomes with citalopram for depression using measurement-based care in STAR*D: implications for clinical practice. Am J Psychiatry. 2006;163:28-40.

5. Kautzky A, Baldinger-Melich P, Kranz GS, Vanicek T, Souery D, Montgomery S, et al. A new prediction model for evaluating treatment-resistant depression. J Clin Psychiatry. 2017;78:215-22.

6. Fu CH, Steiner H, Costafreda SG. Predictive neural biomarkers of clinical response in depression: a meta-analysis of functional and structural neuroimaging studies of pharmacological and psychological therapies. Neurobiol Dis. 2013;52:75-83.

7. Chi KF, Korgaonkar M, Grieve SM. Imaging predictors of remission to anti-depressant medications in major depressive disorder. J Affect Disord. 2015;186:134-44. 
8. Gyurak A, Patenaude B, Korgaonkar MS, Grieve SM, Williams LM, Etkin A. Frontoparietal activation during response inhibition predicts remission to antidepressants in patients with major depression. Biol Psychiatry. 2016;79:274-81.

9. Wagner G, Koch K, Schachtzabel C, Sobanski T, Reichenbach JR, Sauer $\mathrm{H}$, et al. Differential effects of serotonergic and noradrenergic antidepressants on brain activity during a cognitive control task and neurofunctional prediction of treatment outcome in patients with depression. J Psychiatry Neurosci. 2010;35:247-57.

10. Lopez-Sola M, Pujol J, Hernandez-Ribas R, Harrison BJ, Contreras-Rodriguez O, Soriano-Mas C, et al. Effects of duloxetine treatment on brain response to painful stimulation in major depressive disorder. Neuropsychopharmacol: Off Publ Am Coll Neuropsychopharmacol. 2010;35:2305-17.

11. Hahn A, Kranz GS, Seidel EM, Sladky R, Kraus C, Kublbock M, et al. Comparing neural response to painful electrical stimulation with functional MRI at 3 and 7 T. NeuroImage. 2013;82:336-43.

12. Seidel EM, Pfabigan DM, Hahn A, Sladky R, Grahl A, Paul K, et al. Uncertainty during pain anticipation: the adaptive value of preparatory processes. Hum Brain Mapp. 2015;36:744-55.

13. Hahn A, Haeusler D, Kraus C, Hoflich AS, Kranz GS, Baldinger $\mathrm{P}$, et al. Attenuated serotonin transporter association between dorsal raphe and ventral striatum in major depression. Hum Brain Mapp. 2014;35:3857-66.

14. Grieve KL, Acuna C, Cudeiro J. The primate pulvinar nuclei: vision and action. Trends Neurosci. 2000;23:35-39.

15. Langenecker SA, Kennedy SE, Guidotti LM, Briceno EM, Own LS, Hooven T, et al. Frontal and limbic activation during inhibitory control predicts treatment response in major depressive disorder. Biol Psychiatry. 2007;62:1272-80.

16. Phillips ML, Chase HW, Sheline YI, Etkin A, Almeida JR, Deckersbach $\mathrm{T}$, et al. Identifying predictors, moderators, and mediators of antidepressant response in major depressive disorder: neuroimaging approaches. Am J Psychiatry. 2015;172:124-38.

17. Pizzagalli DA. Frontocingulate dysfunction in depression: toward biomarkers of treatment response. Neuropsychopharmacol. 2011;36:183-206.

18. Trattnig S, Springer E, Bogner W, Hangel G, Strasser B, Dymerska B et al. Key clinical benefits of neuroimaging at 7T. Neuroimage. 2016;pii: S1053-8119(16).

19. Marques JP, Norris DG. How to choose the right MR sequence for your research question at 7T and above? Neuroimage. 2017;pii: S1053-8119(17)30341-5.

20. Crane NA, Jenkins LM, Bhaumik R, Dion C, Gowins JR, Mickey $\mathrm{BJ}$, et al. Multidimensional prediction of treatment response to antidepressants with cognitive control and functional MRI. Brain. 2017;140:472-86.

21. Kambeitz J, Cabral C, Sacchet MD, Gotlib IH, Zahn R, Serpa $\mathrm{MH}$, et al. Detecting Neuroimaging Biomarkers for Depression: A Meta-analysis of Multivariate Pattern Recognition Studies. Biol Psychiatry. 2017;82:330-38.

22. Su L, Cai Y, Xu Y, Dutt A, Shi S, Bramon E. Cerebral metabolism in major depressive disorder: a voxel-based meta-analysis of positron emission tomography studies. BMC Psychiatry. 2014;14:321.

23. Hamilton JP, Etkin A, Furman DJ, Lemus MG, Johnson RF, Gotlib IH. Functional neuroimaging of major depressive disorder: a meta-analysis and new integration of base line activation and neural response data. Am J Psychiatry. 2012;169:693-703.

24. Fitzgerald PB, Laird AR, Maller J, Daskalakis ZJ. A meta-analytic study of changes in brain activation in depression. Hum Brain Mapp. 2008;29:683-95.

25. Holthoff VA, Beuthien-Baumann B, Zundorf G, Triemer A, Ludecke S, Winiecki $P$, et al. Changes in brain metabolism associated with remission in unipolar major depression. Acta Psychiatr Scand. 2004;110:184-94.

26. Leaver AM, Espinoza R, Pirnia T, Joshi SH, Woods RP, Narr KL. Modulation of intrinsic brain activity by electroconvulsive therapy in major depression. Biol Psychiatry Cogn Neurosci Neuroimaging. 2016;1:77-86.

27. Greicius MD, Flores BH, Menon V, Glover GH, Solvason HB, Kenna $\mathrm{H}$, et al. Resting-state functional connectivity in major depression: abnormally increased contributions from subgenual cingulate cortex and thalamus. Biol Psychiatry. 2007;62:429-37.

28. Anand A, Li Y, Wang Y, Wu J, Gao S, Bukhari L, et al. Antidepressant effect on connectivity of the mood-regulating circuit: an FMRI study. Neuropsychopharmacol. 2005;30:1334-44.

29. Rose EJ, Simonotto E, Spencer EP, Ebmeier KP. The effects of escitalopram on working memory and brain activity in healthy adults during performance of the n-back task. Psychopharmacol. 2006;185:339-47.

30. Anderson IM, Del-Ben CM, McKie S, Richardson P, Williams SR, Elliott R, et al. Citalopram modulation of neuronal responses to aversive face emotions: a functional MRI study. Neuroreport. 2007;18:1351-55.

31. Frodl T, Scheuerecker J, Schoepf V, Linn J, Koutsouleris N, Bokde AL, et al. Different effects of mirtazapine and venlafaxine on brain activation: an open randomized controlled fMRI study. $\mathbf{J}$ Clin Psychiatry. 2011;72:448-57.

32. Hoflich A, Hahn A, Kublbock M, Kranz GS, Vanicek T, Windischberger C, et al. Ketamine-induced modulation of the thalamocortical network in healthy volunteers as a model for schizophrenia. Int J Neuropsychopharmacol. 2015;18(9).

33. Li X, Nahas Z, Kozel FA, Anderson B, Bohning DE, George MS. Acute left prefrontal transcranial magnetic stimulation in depressed patients is associated with immediately increased activity in prefrontal cortical as well as subcortical regions. Biol Psychiatry. 2004:55:882-90.

34. Pessoa L, Adolphs R. Emotion processing and the amygdala: from a 'low road' to 'many roads' of evaluating biological significance. Nat Rev Neurosci. 2010;11:773-83.

35. Morris JS, Ohman A, Dolan RJ. A subcortical pathway to the right amygdala mediating "unseen" fear. Proc Natl Acad Sci USA. 1999;96:1680-5.

36. Ward R, Danziger S, Owen V, Rafal R. Deficits in spatial coding and feature binding following damage to spatiotopic maps in the human pulvinar. Nat Neurosci. 2002;5:99-100.

37. Krause T, Brunecker P, Pittl S, Taskin B, Laubisch D, Winter B, et al. Thalamic sensory strokes with and without pain: differences in lesion patterns in the ventral posterior thalamus. J Neurol Neurosurg Psychiatry. 2012;83:776-84.

38. Beck AT. The evolution of the cognitive model of depression and its neurobiological correlates. Am J Psychiatry. 2008;165:969-77.

39. Disner SG, Beevers CG, Haigh EA, Beck AT. Neural mechanisms of the cognitive model of depression. Nat Rev Neurosci. 2011;12:467-77.

40. Gex-Fabry M, Gervasoni N, Eap CB, Aubry JM, Bondolfi G, Bertschy G. Time course of response to paroxetine: influence of plasma level. Prog Neuropsychopharmacol Biol Psychiatry. 2007;31:892-900.

41. Drevets WC, Price JL, Furey ML. Brain structural and functional abnormalities in mood disorders: implications for neurocircuitry models of depression. Brain Struct Funct. 2008;213: 93-18.

42. Godlewska BR, Browning M, Norbury R, Cowen PJ, Harmer CJ. Early changes in emotional processing as a marker of clinical response to SSRI treatment in depression. Transl Psychiatry. 2016;6:e957.

43. Spies M, Kraus C, Geissberger N, Auer B, Klobl M, Tik M, et al. Default mode network deactivation during emotion processing 
predicts early antidepressant response. Transl Psychiatry. 2017;7: e1008.

44. Fox MD, Corbetta M, Snyder AZ, Vincent JL, Raichle ME. Spontaneous neuronal activity distinguishes human dorsal and ventral attention systems. Proc Natl Acad Sci USA. 2006;103: 10046-51.

45. Downar J, Crawley AP, Mikulis DJ, Davis KD. A multimodal cortical network for the detection of changes in the sensory environment. Nat Neurosci. 2000;3:277-83.

46. Molenberghs P, Johnson H, Henry JD, Mattingley JB. Understanding the minds of others: a neuroimaging meta-analysis. Neurosci Biobehav Rev. 2016;65:276-91.

47. Bora E, Berk M. Theory of mind in major depressive disorder: a meta-analysis. J Affect Disord. 2016;191:49-55.

48. Bertoux M, Delavest M, de Souza LC, Funkiewiez A, Lepine JP, Fossati $\mathrm{P}$, et al. Social cognition and emotional assessment differentiates frontotemporal dementia from depression. J Neurol Neurosurg Psychiatry. 2012;83:411-16.

49. Moratti S, Rubio G, Campo P, Keil A, Ortiz T. Hypofunction of right temporoparietal cortex during emotional arousal in depression. Arch Gen Psychiatry. 2008;65:532-41.

50. Domschke K, Zwanzger P, Rehbein MA, Steinberg C, Knoke K, Dobel C, et al. Magnetoencephalographic correlates of emotional processing in major depression before and after pharmacological treatment. Int J Neuropsychopharmacol. 2015; 19.

51. Decety J, Lamm C. The role of the right temporoparietal junction in social interaction: how low-level computational processes contribute to meta-cognition. Neuroscientist. 2007;13:580-93.

52. McMillan CT, Avants BB, Cook P, Ungar L, Trojanowski JQ, Grossman $\mathrm{M}$. The power of neuroimaging biomarkers for screening frontotemporal dementia. Hum Brain Mapp. 2014;35:4827-40.

53. Quiton RL, Keaser ML, Zhuo J, Gullapalli RP, Greenspan JD. Intersession reliability of fMRI activation for heat pain and motor tasks. Neuroimage Clin. 2014;5:309-21.
54. Upadhyay J, Lemme J, Anderson J, Bleakman D, Large T, Evelhoch JL, et al. Test-retest reliability of evoked heat stimulation BOLD fMRI. J Neurosci Methods. 2015;253:38-46.

55. Raemaekers M, du Plessis S, Ramsey NF, Weusten JM, Vink M. Test-retest variability underlying fMRI measurements. NeuroImage. 2012;60:717-27.

56. Gorgolewski KJ, Storkey AJ, Bastin ME, Whittle I, Pernet C. Single subject fMRI test-retest reliability metrics and confounding factors. NeuroImage. 2013;69:231-43.

57. McGrath CL, Kelley ME, Holtzheimer PE, Dunlop BW, Craighead WE, Franco AR, et al. Toward a neuroimaging treatment selection biomarker for major depressive disorder. JAMA Psychiatry. 2013;70:821-29.

58. McGrath CL, Kelley ME, Dunlop BW, Holtzheimer PE 3rd, Craighead WE, Mayberg HS. Pretreatment brain states identify likely nonresponse to standard treatments for depression. Biol Psychiatry. 2014;76:527-35.

59. Kasper S, Corruble E, Hale A, Lemoine P, Montgomery SA, Quera-Salva MA. Antidepressant efficacy of agomelatine versus SSRI/SNRI: results from a pooled analysis of head-to-head studies without a placebo control. Int Clin Psychopharmacol. 2013;28:12-19.

60. Kasper S. Efficacy of antidepressants in the treatment of severe depression: the place of mirtazapine. J Clin Psychopharmacol. 1997;17:19S-8S.

61. Williams LM, Rush AJ, Koslow SH, Wisniewski SR, Cooper NJ, Nemeroff $\mathrm{CB}$, et al. International Study to Predict Optimized Treatment for Depression (iSPOT-D), a randomized clinical trial: rationale and protocol. Trials. 2011;12:4.

62. Williams LM, Korgaonkar MS, Song YC, Paton R, Eagles S, Goldstein-Piekarski A, et al. Amygdala reactivity to emotional faces in the prediction of general and medicationspecific responses to antidepressant treatment in the randomized iSPOT-D trial. Neuropsychopharmacol. 2015;40: 2398-08. 